\title{
THE
}

\section{PFAS fluidize synthetic and bacterial lipid monolayers based on hydrophobicity and lipid charge}

\author{
Aleksandra Naumann \\ University of Rhode Island \\ Jessica Alesio \\ University of Rhode Island \\ Monika Poonia \\ University of Rhode Island \\ Geoffrey D. Bothun \\ University of Rhode Island, gbothun@uri.edu
}

Follow this and additional works at: https://digitalcommons.uri.edu/che_facpubs

\section{The University of Rhode Island Faculty have made this article openly available.}

Please let us know how Open Access to this research benefits you.

This is a pre-publication author manuscript of the final, published article.

Terms of Use

This article is made available under the terms and conditions applicable towards Open Access

Policy Articles, as set forth in our Terms of Use.

\section{Citation/Publisher Attribution}

Maumann, A., Alesio, J., Poonia, M., \& Bothun, G. D. (2022). PFAS fluidize synthetic and bacterial lipid monolayers based on hydrophobicity and lipid charge. Journal of Environmental Chemical Engineering, 10(2), 107351. https://doi.org/10.1016/j.jece.2022.107351

Available at: https://doi.org/10.1016/j.jece.2022.107351 


\section{$1 \quad$ PFAS fluidize synthetic and bacterial lipid}

2 monolayers based on hydrophobicity and lipid

3 charge

4

5 Aleksandra Naumann ${ }^{1}$, Jessica Alesio ${ }^{1}$, Monika Poonia ${ }^{l}$, and Geoffrey D. Bothun ${ }^{l, *}$

6 Department of Chemical Engineering, University of Rhode Island, 2 East Alumni Ave, Kingston,

$7 \quad$ RI, 02881

8 *Corresponding Author. Tel: +1-401-874-9517, Fax: +1-401-874-2656, E-mail:

9 gbothun@uri.edu

10

11 KEYWORDS: Perfluoroalkyl substance; perfluorooctanoic acid; lipid monolayer; surface

12 pressure; bacteria membrane

14 Abstract

Poly- and Perfluoroalkyl substances (PFASs) are pollutants of emerging concern

16 that persist in nature and pose environmental health and safety risks. PFAS disrupt biological

17 membranes resulting in cellular inhibition, but the mechanism of disruption and the role of lipid

18 composition remain unclear. We examine the role of phospholipid saturation and headgroup

19 charge on the interactions between PFASs and phospholipid monolayers comprised of synthetic

20 phosphocholine (PC) and phosphoglycerol (PG) lipids and prepared from bacteria membrane

21 extracts rich in PG lipids from an environmentally relevant marine bacterium Alcanivorax 
22 borkumensis. When deposited on a buffered subphase containing PFAS, PFAS mixed within and

23 fluidized zwitterionic and net-anionic monolayers leading to increases in monolayer

24 compressibility that were driven by a combination of PFAS hydrophobicity and monolayer

25 charge density. Differences in the monolayer response using saturated or unsaturated lipids are

26 attributed to the ability of the unsaturated lipids to accommodate PFAS within 'void space'

27 arising from the bent lipid tails. Similar fluidization and compressibility behavior were also

28 observed in A. borkumensis lipid monolayers. This work provides new insight into PFAS

29 partitioning into bacterial membranes and the effect PFAS have on the physicomechanical

30 properties of zwitterionic and charged lipid monolayers.

\section{1. Introduction}

Poly- and Perfluoroalkyl substances (PFAS) are pollutants of emerging concern that

34 exhibit an unprecedented ability to accumulate within the environment. ${ }^{1-3}$ PFAS, which are

35 fluorinated amphiphiles with low volatility, have been used in a wide range of products and

36 processes where low adhesion and water and oil repellency are required. ${ }^{4,5}$ These properties also

37 cause PFAS to persist in the environment and bioaccumulate. For example, one of the most-

38 studied PFAS, perfluorooctanesulfonic acid (PFOS) is known to be present in blood serum of US

39 citizens at concentrations near $40 \mathrm{ng} / \mathrm{mL} .{ }^{6}$ In addition, the short-chain PFAS

40 perfluorobutanesulfonic acid (PFBS) found in cord blood was positively associated with

41 preeclampsia. ${ }^{7}$ Even years after exposure, high levels of PFAS remain in the body. ${ }^{8,9}$ Although

42 there has been a shift to PFAS with shorter fluorinated tails that are thought to bioaccumulate to

43 a lesser extent than longer PFAS, compounds with long fluorinated tails $\left(\mathrm{C}_{\mathrm{nF}} \geq 7\right)$ remain in the

44 environment, even in remote areas such as the arctic through the global water cycle. ${ }^{6,10,11}$ 
PFAS bioaccumulation has been linked to lipid and protein binding mechanisms,

46 consistent with the hydrophobic nature of the compounds. ${ }^{12-15}$ While there have been numerous

47 studies focused on PFAS-protein binding, comparably few studies have focused on PFAS

48 partitioning into lipid bilayer membranes or monolayers. ${ }^{16-22}$ Perfluorooctanoic acid (PFOA) and

49 PFOS, legacy eight-carbon PFASs, have been shown to readily partition into bilayers comprised

50 of zwitterionic phospholipids, disrupting inter-lipid interactions and disordering the

51 bilayer. ${ }^{16,21,22}$ Interestingly, PFOA partitioning was dependent on phospholipid chain length ${ }^{22}$

52 while PFOS partitioning was not, ${ }^{16}$ which may reflect the greater hydrophobicity of PFOS. Even

53 short chain PFAS such as PFBS partition into and disrupt zwitterionic phospholipid bilayers. ${ }^{17,18}$

54 Phospholipid monolayer studies provide additional insight into PFAS partitioning and its

55 effects on physicomechanical monolayer properties. PFOA, PFOS and PFBS have been shown to

56 partition into zwitterionic phospholipid monolayers. ${ }^{18-20}$ As the monolayers were compressed,

57 packing the lipids more closely together and increasing the inter-lipid interactions, PFOA was

58 expelled from the monolayer while the sulfonic acids $\operatorname{PFOS}^{19,20}$ and $\operatorname{PFBS}^{18}$ were retained.

59 Collectively, previous work such as these show that bilayer and monolayer studies provide

60 complimentary information that can be combined to better understand how lipid partitioning

61 varies with PFAS and lipid composition.

62 Bacteria have a central function in ecosystems, and it is critical to understand how PFAS

63 partition into and disrupt bacterial cell membranes. Previous studies have shown that PFAS

64 partitioning into Staphylococcus epidermidis and Aliivibrio fischeri was dependent on PFAS

65 hydrophobicity, ${ }^{23}$ determined largely by fluorinated tail length. While the general behavior for

66 bacteria partitioning with PFAS hydrophobicity agreed with partitioning observed with

67 zwitterionic phospholipid bilayers, lower PFAS partition coefficients for bacteria were attributed 
68 to electrostatic repulsion between PFASs and the negatively charged bacterial membrane. With

69 A. fischeri the membranes become more permeable after PFAS exposure, which may have

70 contributed to increased quorum sensing. ${ }^{24}$ PFOA and PFOS have also been shown to disrupt

71 Escherichia coli membranes, contributing to toxicity ${ }^{25}$ and increasing biofilm formation as a

72 stress response to the added PFAS. ${ }^{26}$

73 The objective of this work was twofold; (1) to examine the effect of phospholipid tail

74 saturation and charge on PFAS interactions within monolayers and (2) to determine if these

75 interactions are also observed in monolayers comprised of lipids extracted from Alcanivorax

76 borkumensis. Saturated and mono-unsaturated phosphocholine (PC) and phosphoglycerol (PG)

77 lipids were chosen to represent bacterial lipids. A. borkumensis was employed as a model

78 organism. It is a ubiquitous marine bacterium known for its ability to utilize alkanes as a carbon

79 source and is often found to be a dominant species in association with marine oil spills. ${ }^{27,28}$ The

80 primary lipid fatty acids of $A$. borkumensis grown on the same carbon source employed in this

81 work - saturated $\mathrm{C}_{16}$ tails $(16: 0, \sim 30 \%)$ and mono-unsaturated $\mathrm{C}_{18}$ tails $(18: 1 \Delta 9-\text { cis, } \sim 40 \%)^{29}-$

82 are similar to the tail structures used in the monolayer studies. The results show that phenomena

83 observed in synthetic lipid monolayers also describe PFAS interactions in complex bacterial

84 membrane extracts.

85

\section{2. Experimental}

87 2.1. Chemicals

Perfluorooctanoic acid (PFOA), perfluorononanoic acid (PFNA), perfluorooctanesulfonic

89 acid (PFOS, potassium salt), and perfluorohexanesulfonic acid (PFHxS, sodium salt) were

90 purchased from AccuStandard ${ }^{\circledR}($ New Haven, CT) with purities $>96 \%$. The phospholipids $1,2-$ 
91 dimyristoyl-sn-glycero-3-phosphocholine (DMPC), 1,2-dimyristoyl-sn-glycero-3-

92 phosphoglycerol (DMPG, sodium salt), 1,2-dioleoyl-sn-glycero-3-phosphocholine (DOPC), and

93 1,2-dioleoyl-sn-glycero-3-phosphoglycerol (DOPG, sodium salt) were purchased from Avanti ${ }^{\circledR}$

94 Polar Lipids (Alabaster, AL). All the materials were used as received without further

95 purification. Experiments were conducted in $10 \mathrm{mM} \mathrm{N}$-(2-hydroxyethyl)piperazine- $\mathrm{N}^{\prime}-(2-$

96 ethanesulfonic acid) (HEPES, Sigma-Aldrich) buffer at $\mathrm{pH} 7$ prepared using sterile, ultrafiltered

97 deionized water (DIW) obtained from a Millipore Direct-3Q purification system. Molecular

98 properties of the PFAS and phospholipids - the number of carbons $\left(\mathrm{C}_{\mathrm{nF}}\right.$ and $\mathrm{C}_{\mathrm{n}}$ :cis $)$, charge,

99 octanol/water partition coefficient $\left(\log \mathrm{K}_{\mathrm{ow}}\right)$, van der Waals volume $\left(\mathrm{V}_{\mathrm{vdw}}\right)$, and polar surface

100 area $\left(\mathrm{A}_{\text {polar }}\right)$ - are summarized in Table 1.

101

102 Table 1. Molecular properties of PFAS and phospholipid compounds.

\begin{tabular}{|c|c|c|c|c|c|}
\hline Compound & $\mathrm{C}_{\mathrm{nF}}\left(\mathrm{C}_{\mathrm{n}}\right)^{\mathrm{b}}$ & Charge & $\log K_{o w}{ }^{d}$ & $\mathrm{~V}_{\mathrm{vdw}}\left(\AA^{3}\right)^{\mathrm{e}}$ & $A_{\text {polar }}\left(\AA^{3}\right)^{\mathrm{e}}$ \\
\hline \multicolumn{6}{|l|}{ PFASs } \\
\hline PFOA & $7(8)$ & - & $3.10(5.68)$ & 231 & 40 \\
\hline PFNA $^{a}$ & $8(9)$ & - & $3.54(6.51)$ & 258 & 40 \\
\hline PFHxS & $6(6)$ & - & $2.20(3.39)$ & 221 & 57 \\
\hline \multirow{2}{*}{ PFOS } & $8(8)$ & - & $5.61(5.77)$ & 275 & 57 \\
\hline & $\mathrm{C}_{\mathrm{n}}: \mathrm{cis}^{\mathrm{c}}$ & Charge & & $\mathrm{V}_{\mathrm{vdw}}\left(\AA^{3}\right)^{\mathrm{e}}$ & $\mathrm{A}_{\text {polar }}\left(\AA^{3}\right)^{\mathrm{e}}$ \\
\hline \multicolumn{6}{|l|}{ Lipids } \\
\hline DMPC & 14:0 & $-/+$ & & 716 & 111 \\
\hline DMPG & 14:0 & - & & 682 & 152 \\
\hline DOPC & 18:1 & $-/+$ & & 836 & 111 \\
\hline DOPG & $18: 1$ & - & & 803 & 152 \\
\hline
\end{tabular}

${ }^{\mathrm{a}}$ Restricted to bacterial lipid monolayer studies.

$104{ }^{b} C_{n F}=$ number of fluorinated carbons; $C_{n}=$ total number of carbons.

$105{ }^{\mathrm{c}} \mathrm{C}_{\mathrm{n}}$ :cis = ratio of the total number of carbons to the number of double bonds in the tails.

$106{ }^{\mathrm{d}} \mathrm{K}_{\mathrm{ow}}=\mathrm{octanol} /$ water partition coefficient, experimental average (predicted average), US EPA

107 CompTox Chemicals Dashboard. ${ }^{30}$

$108{ }^{\mathrm{e}}$ Computed with Marvin Sketch (Version 20.11).

109 
112 provided in Supplementary Material for bacteria growth and lipid extraction. Briefly, bacteria

113 were grown in sterile $125 \mathrm{~mL}$ baffled Erlenmeyer flasks for $72 \mathrm{~h}$ on the platform shaker at

114 ambient temperature. ${ }^{27}$ Cells were grown to an optical density, $\mathrm{OD}_{600}$, of 1.2 and visually

115 examined under a microscope (Figure S1). The bacteria were centrifuged at $7000 \mathrm{~g}$ for $30 \mathrm{~min}$ to

116 form a pellet, which was then resuspended and washed twice with artificial seawater via

117 centrifugation. For the final resuspension, the bacteria were pelletized again and resuspended in

$1182 \mathrm{~mL} 0.9 \% \mathrm{NaCl}(\mathrm{w} / \mathrm{w})$. Lipid extraction was achieved using a modified Bligh and Dyer

119 method. ${ }^{31,32}$ A total of 9 pellets (9 bacteria cultures) yielded an average of $3.52 \pm 0.74 \mathrm{mg}$ of

120 extracted lipids per pellet, which were pooled for the monolayer studies (Figure S2, S3; Table

121 S1).

122

123 2.3. Surface pressure measurements and Langmuir isotherms

125 Scientific) measuring $364 \times 75 \times 4 \mathrm{~mm}(\mathrm{~L} \times \mathrm{W} \times \mathrm{H})$ with a total working surface area of $240 \mathrm{~cm}^{2}($ see

126 Figure S4 for an overview). The trough was placed on an air table (model Onyx 7A, Herzan,

127 Laguna Hills, CA) for passive vibration control. All experiments were conducted at $20{ }^{\circ} \mathrm{C}$ with a

$12810 \mathrm{mM}$ HEPES aqueous subphase. Prior to forming a Langmuir monolayer, the trough and

129 barriers were rinsed thoroughly with ethanol three times and then the trough was filled with the

130 aqueous subphase. The trough was considered sufficiently clean if the fluctuations in surface

131 pressure at the neat air/water interface were less than $0.3 \mathrm{mN} / \mathrm{m}$ as the barriers were compressed

132 down to $20-30 \%$ of the working surface area. Surface pressure was continuously measured using

133 paper Wilhelmy plates (Nanoscience Instruments, Phoenix, AZ). 
135 chloroform $(1 \mathrm{~g} / \mathrm{L})$ on the surface of the aqueous subphase (with or without added PFAS) using a

$13650 \mu \mathrm{L}$ Hamilton microsyringe. The chloroform was allowed to evaporate over $15 \mathrm{~min}$. The

137 surface pressure-area $(\pi-\overline{\mathrm{A}}$, where $\overline{\mathrm{A}}$ is the mean molecular area or $\pi-\mathrm{A}$, where $\mathrm{A}$ is the total

138 trough area) isotherms were recorded by compressing the monolayers with a barrier speed of 3

$139 \mathrm{~mm} / \mathrm{min}$, which allowed the monolayer to reach a pseudo-equilibrium condition. Surface

140 pressure was recorded every 10 s using an integrated balance. $\bar{A}$ was calculated based on the

141 total number of lipid molecules deposited at the air/water interface divided by the initial area of

142 the trough. Brewster Angle Microscope (MicroBAM, KSV NIMA, Biolin Scientific) was used to

143 image the monolayers in situ.

144 Compressibility moduli, $C_{s}{ }^{-1}$, or the reciprocal of the compressibility, were calculated

145 from the monolayer isotherms according to equation 1.

$$
C_{s}^{-1}=-\bar{A} \frac{d \pi}{d \bar{A}}
$$

$147 C_{s}^{-1}$ describes how resistant a monolayer is to compression; high values of $C_{s}^{-1}$ correspond to a 148 rigid monolayer with high resistance to compression, while low values of $C_{s}{ }^{-1}$ correspond to a 149 fluid monolayer that requires less force to compress. The equation can also be applied when the 150 area is the total trough area, A.

151 The surface activity of PFAS (no lipid present) were examined under compression by 152 adding PFAS to the subphase and allowing the system to equilibrate for $15 \mathrm{~min}$. PFAS 153 monolayers were then compressed and recorded as they were for lipid monolayers. Lipid 154 isotherms and PFAS surface pressure measurements were conducted in triplicate and the results 155 are presented as the mean surface pressure. For ease of viewing, symbols were added 
156 representing the mean value at every 50th data point. Standard error is shown in all figures as a

157 lighter colored band around the mean.

\section{Results and Discussion}

Surface pressure-area $(\pi-\mathrm{A})$ measurements for PFAS were generated to determine their 161 surface activity at a bulk subphase concentration of $10^{-4} \mathrm{M}$ (Figure S5). PFAS are soluble in 162 water and upon compression the results reflect a competition between PFAS packing at the 163 air/water interface and the adsorption energy. PFOA exhibited an s-shaped curve with $\pi$ 164 increasing from 4.2 to $11.5 \mathrm{mN} / \mathrm{m}$ with compression as PFOA packed more tightly at the 165 interface and reduced the interfacial tension. PFOS exhibited a flat curve with comparatively 166 high $\pi$ between $\sim 14-16 \mathrm{mN} / \mathrm{m}$. In this case the surface was saturated with PFOS and the results

167 suggests that PFOS molecules were expelled from the interface during compression, maintaining 168 a near-constant surface pressure. The higher surface activity of PFOS compared to PFOA is 169 consistent with PFOS being more hydrophobic based on $\log \mathrm{K}_{\mathrm{ow}}$ (Table 1; comparison of 170 experimental averages). ${ }^{33}$ The range of $\pi$ at the same PFAS concentrations are similar to 171 previous work. ${ }^{34,35}$ Estimated effective mean PFAS molecular areas $\left(\overline{\mathrm{A}}_{\mathrm{P}}\right)$ were extrapolated from

172 Schaefer et $\mathrm{al}^{34}$ and Costanza et $\mathrm{al}^{35}$ and are compared to computed minimum and maximum 173 molecular area projections (Table 2). This comparison suggests that PFOS formed a packed 174 monolayer oriented perpendicular to the air/water interface while PFOA formed a sparser 175 monolayer with a higher degree of parallel orientation.

177 Table 2. Estimated and computed effective mean PFAS molecular areas.

\begin{tabular}{cccc}
\hline Compound & Estimated $\bar{A}_{P}\left(\AA^{2}\right)$ & Computed $\bar{A}_{P, \min }\left(\AA^{2}\right)^{\mathrm{c}}$ & Computed $\bar{A}_{P, \max }\left(\AA^{2}\right)^{\mathrm{c}}$ \\
\hline PFOA & $83^{\mathrm{a}}, 181^{\mathrm{b}}$ & 36 & 67
\end{tabular}


178 ${ }^{\mathrm{a}}$ Extrapolated from Schaefer et al. ${ }^{34}$

179 bxtrapolated from Costanza et al. ${ }^{35}$

$180{ }^{\circ}$ Computed with Marvin Sketch (Version 20.11).

\subsection{Saturated Phospholipid Monolayers (DMPC and DMPC/DMPG)} with and without PFAS as a function of the anionic DMPG mole fraction $\left(\mathrm{X}_{\mathrm{DMPG}}=0,0.15\right.$, and 0.3; Figure 1). With increasing $\mathrm{X}_{\mathrm{DMPG}}$, the isotherms without added PFAS become steeper and 186 phase transitions from liquid-expanded (LE) to liquid-condensed (LC) phases were observed 187 between $25-30 \mathrm{mN} .{ }^{36}$ DMPC itself does not exhibit a LE-LC transition. The lift off pressure, 188 which corresponds to the area per molecule when the surface pressure can be detected, also 189 increased with DMPG concentration $\left(\sim 20 \%\right.$ from $\mathrm{X}_{\mathrm{DMPG}}=0$ to 0.3$)$ due to repulsive electrostatic 190 interactions between DMPG lipids that increased the effective mean lipid molecular area, $\bar{A} \cdot{ }^{37}$ 191 With PFAS present in the subphase, the isotherms showed an increase in $\pi$ after

192 formation and equilibration at the highest mean molecular areas, $\bar{A}$, reflecting the presence of 193 PFAS at the interface. The effect was most pronounced for PFOS, which showed the greatest 194 surface activity (Figure 1a-c). The presence of anionic PFAS added to the electrostatic repulsion 195 within the monolayers originating with DMPG and altered the inter-lipid interactions that led to 196 the LE-LC phase transition. 

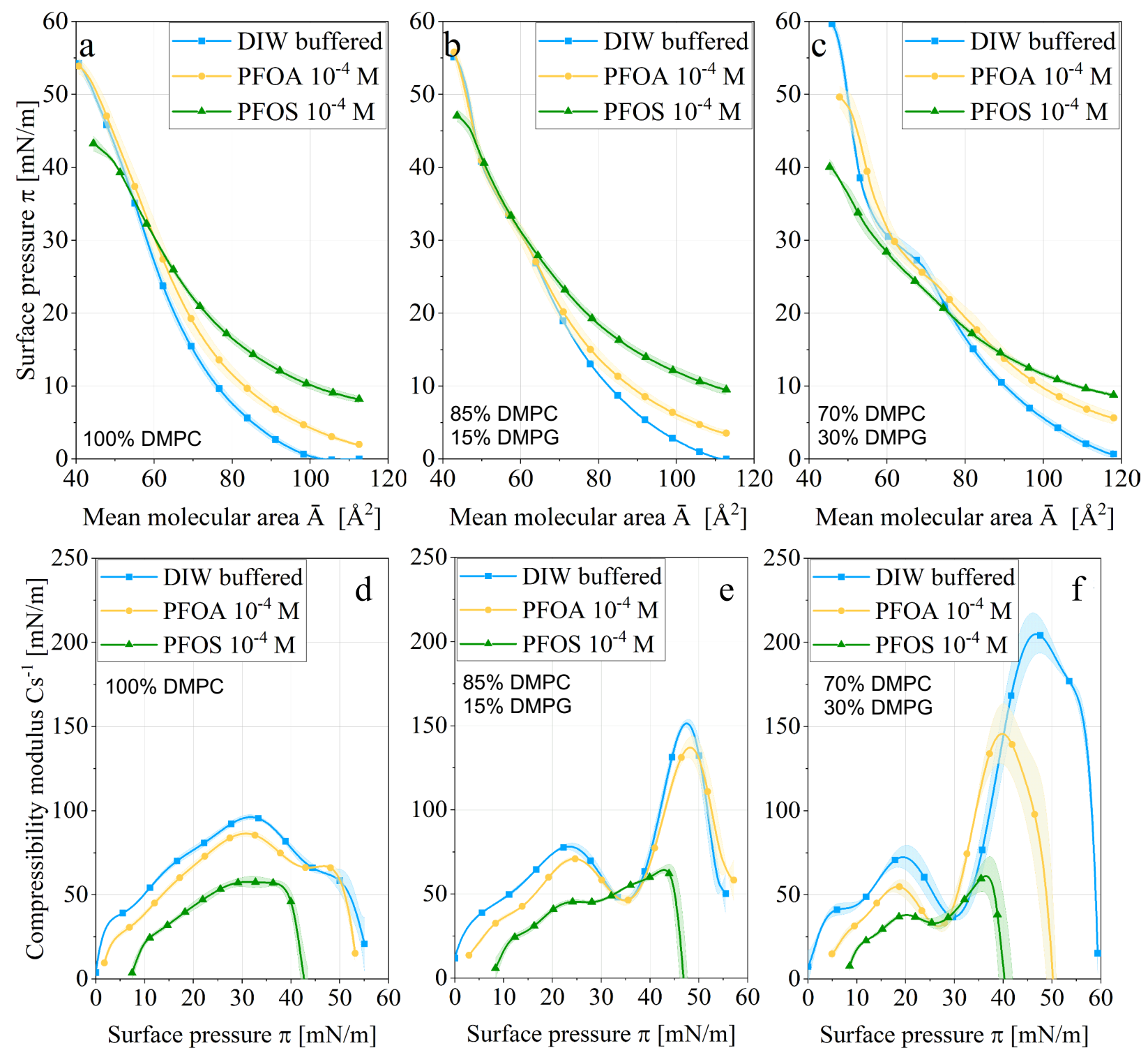

199 Figure 1. Surface pressure-area isotherms, $\pi-\overline{\mathrm{A}}$ (a-c), and compressibility moduli, $\mathrm{C}_{\mathrm{s}}{ }^{-1}$ (d-f), for

200 DMPC monolayers as a function of DMPG concentration ( $\left.\mathrm{X}_{\mathrm{DMPG}}\right)$ in the absence (blue squares)

201 or presence of $10^{-4} \mathrm{M}$ PFOA (yellow circles) or PFOS (green triangles) in a HEPES buffered

202 DIW subphase. a, d) $\mathrm{X}_{\mathrm{DMPG}}=0$; $\left.\mathrm{b}, \mathrm{e}\right) \mathrm{X}_{\mathrm{DMPG}}=0.15$; and c, f) $\mathrm{X}_{\mathrm{DMPG}}=0.3$. The colored bands

203 shown in a-f for each condition represent the standard error of three independent experiments. 
The isotherms with added PFAS were less steep upon compression, implying a more compressible monolayer, and intersected the phospholipid isotherms. Results for the

207 compressibility moduli, $\mathrm{C}_{\mathrm{s}}^{-1}$, are shown in Figure $1 \mathrm{~d}-\mathrm{f}$. The local minima near $\sim 30-35 \mathrm{mN} / \mathrm{m}$ for $208 \mathrm{X}_{\mathrm{DMPG}}$ at 0.15 and 0.3 represent $\pi$ at the midpoint of the LE-LC phase transition. The minima are 209 followed by rapid increases in $\mathrm{C}_{\mathrm{s}}^{-1}$ (decreases in compressibility) as the structured LC phase 210 formed. ${ }^{20}$ Increasing DMPG concentrations led to decreases in $\mathrm{C}_{\mathrm{s}}{ }^{-1}$ when the monolayers were in 211 the LE phase (more compressible) and increases $\mathrm{C}_{\mathrm{s}}^{-1}$ in the LC phase (less compressible) at high 212 surface pressures. This behavior is tied to DMPG, which reduced lipid packing in the LE phase 213 via electrostatic repulsion and drove the LE-LC phase transition. The addition of PFAS to the 214 subphase led to overall decreases in the compressibility moduli, with the decreases being more 215 pronounced with increasing DMPG concentration. PFOS had a greater influence on $\mathrm{C}_{\mathrm{s}}{ }^{-1}$ because 216 it is larger and more hydrophobic (Table 1, reflected in $\mathrm{C}_{\mathrm{nF}}$ ), and has greater surface activity than 217 PFOA.

219 the compressibility moduli. Only at the highest DMPG mole fraction, $\mathrm{X}_{\mathrm{DMPG}}=0.3$ (Figure 1f),

220 did we observe a shift in the transition to lower $\pi$ and $\bar{A}$, consistent with a lipid condensing 221 effect caused by the PFAS. Lipid condensation has been reported Lv and Sun, ${ }^{39}$ where molecular 222 dynamics simulations revealed that saturated 1,2-dipalmitoyl-sn-glycero-3-phosphocholine $223\left(\mathrm{DPPC}, \mathrm{C}_{\mathrm{n}}: \mathrm{cis}=16: 0\right)$ in a bilayer condense around PFOS to shield it from water, yielding a 224 more thermodynamically favorably state. Since DMPC $\left(\mathrm{C}_{\mathrm{n}}\right.$ :cis $\left.=14: 0\right)$ does not exhibit a LE-LC 225 transition alone, we only observed this condensing effect with DMPG was present.

226 To examine the effective area occupied by PFAS initially and the likelihood of PFAS 227 exclusion at high compression, we determined the limiting area at high $\bar{A}$ (LE phase) and the 
228 maximum surface pressure, respectively. The limiting area, expressed as the difference in

229 limiting areas with and without added PFASs $\left(\overline{\mathrm{A}}_{\mathrm{LP}, \min }-\overline{\mathrm{A}}_{\mathrm{L}, \mathrm{min}}\right)$, are shown in Figure 2a. The

230 values increase with DMPG concentration, denoting the role of electrostatic repulsion in

231 monolayer expansion. The values of $\bar{A}_{L P, \min }-\bar{A}_{L, \min }$ are an significantly lower than the estimated

232 and computed PFAS area per headgroup shown in Table 2, suggesting that the PFAS aligned

233 with the lipid tails and/or condensed surrounding lipids to reduce the mean lipid area per

234 molecule.
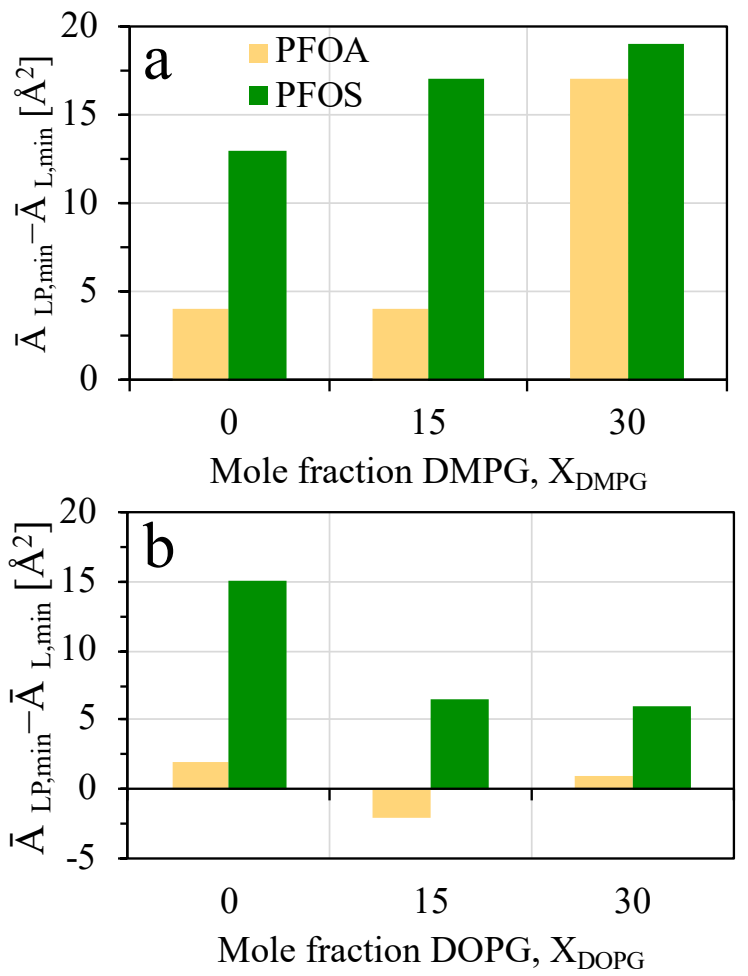

236 Figure 2. Difference in limiting areas with and without added PFOA or PFOS ( $\left.\overline{\mathrm{A}}_{\mathrm{LP}, \min }-\overline{\mathrm{A}}_{\mathrm{L}, \mathrm{min}}\right)$

237 for a) DMPC/DMPG and b) DOPC/DOPG monolayers as a function PG lipid mole fraction.

238 These values were taken from the mean isotherms. 
Maximum surface pressures, $\pi_{\max }$, are shown as a function of PG concentration in Figure

241 3. The labels ' $c$ ' and ' $p$ ' denote whether the maximum refers to a collapse (an abrupt drop in $\pi$

242 with compression at low $\bar{A}$ ) or a plateau, with little to no evidence of collapse, respectively.

243 PFOA did not lower $\pi_{\max }$ at $\mathrm{X}_{\mathrm{DMPG}}=0$ or 0.15 , and at $\mathrm{X}_{\mathrm{DMPG}}=0.3$ the decrease in $\pi_{\max }$ suggests

244 that the phospholipids did not pack tightly enough to 'squeeze' PFOA out of the monolayer due

245 to headgroup repulsion. However, a significant decrease in the collapse and plateau pressures

246 were observed for PFOS. These results indicate that PFOA was partially expelled from the

247 monolayers at high compression while PFOS remains within the monolayers, consistent with

248 PFOS showing greater phospholipid bilayer partitioning than PFOA. ${ }^{2,3}$ The decrease of the

249 collapse pressure with a PFOS-rich subphase can be explained by the comparably high surface

250 activity, which leads to disordering effects due to disrupted inter-phospholipid interactions,

251 namely van der Waals attraction. ${ }^{38}$ This phenomenon is coupled with increased electrostatic

252 repulsion within the monolayer due to PFOS, which restricts compression at low $\overline{\mathrm{A}}$ resulting in

253 collapse. 

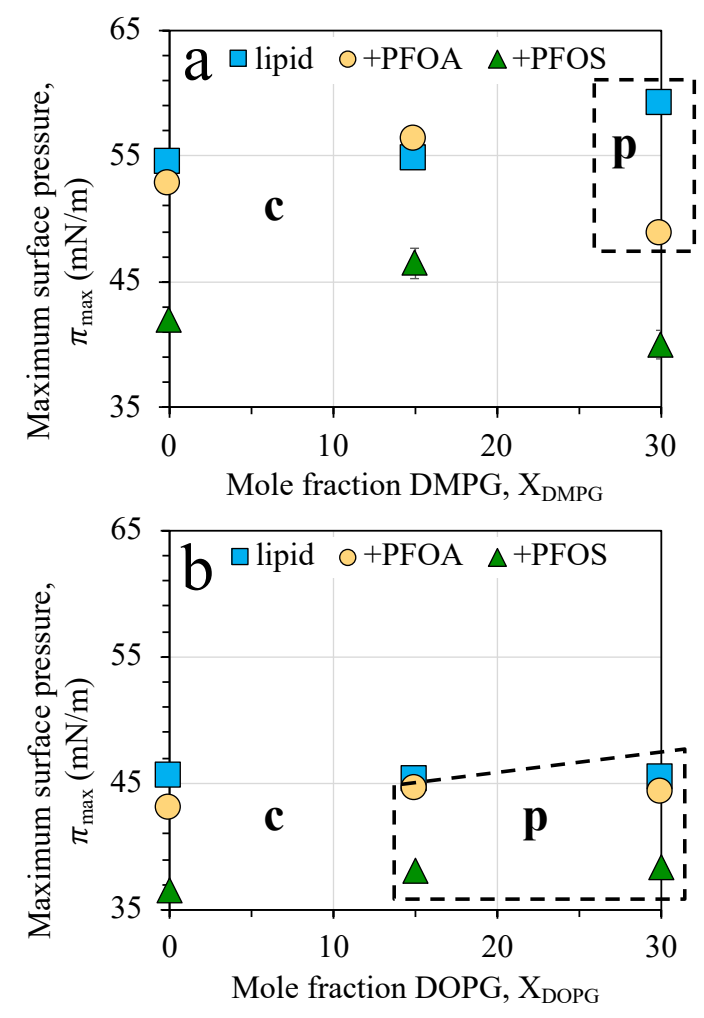

255 Figure 3. Maximum surface pressure, $\pi_{\max }$, as a function of PG lipid concentration for a)

256 DMPC/DMPG and b) DOPC/DOPG with added PFOA or PFOS. The labels 'c' and 'p' denote

257 whether $\pi_{\max }$ refers to a collapse or a plateau (dashed rectangle shows plateau region). Standard

258 error bars are shown based on triplicate measurements. Error bars not visible are smaller than the 259 symbols.

260

261 BAM images of the monolayers compressed to $40 \mathrm{mN} / \mathrm{m}$ with $\mathrm{X}_{\mathrm{PG}}=0$ and 0.3 are shown 262 in Figure 4A. PFOA and PFOS led to the formation of bright 'spots' within DMPC monolayers $263\left(\mathrm{X}_{\mathrm{DMPG}}=0\right)$ indicative of condensed, solid domains that are thicker than the surrounding bulk 264 lipid phase. These solid domains became more prevalent with increasing $\pi$ and were only 265 observed with added PFAS. Phospholipid condensation observed by BAM and by $\bar{A}_{\mathrm{LP}, \min }-$ $266 \bar{A}_{\mathrm{L}, \min }$ (Figure 2a) for DMPC monolayers support this mechanism. At $\mathrm{X}_{\mathrm{DMPG}}=0.3$ condensation 
267 could not be discerned as the solid phases were observed with and without added PFAS.

268 Phospholipid condensation is typically associated with a stiffer or less compressible (higher $\mathrm{C}^{-1}$ )

269 monolayer as opposed to a more compressible monolayer (Figure 1d-f). Thus, PFOA and PFOS

270 have anomalous affects - they condense phospholipid domains while still fluidizing the

271 monolayer and rendering it more compressible.

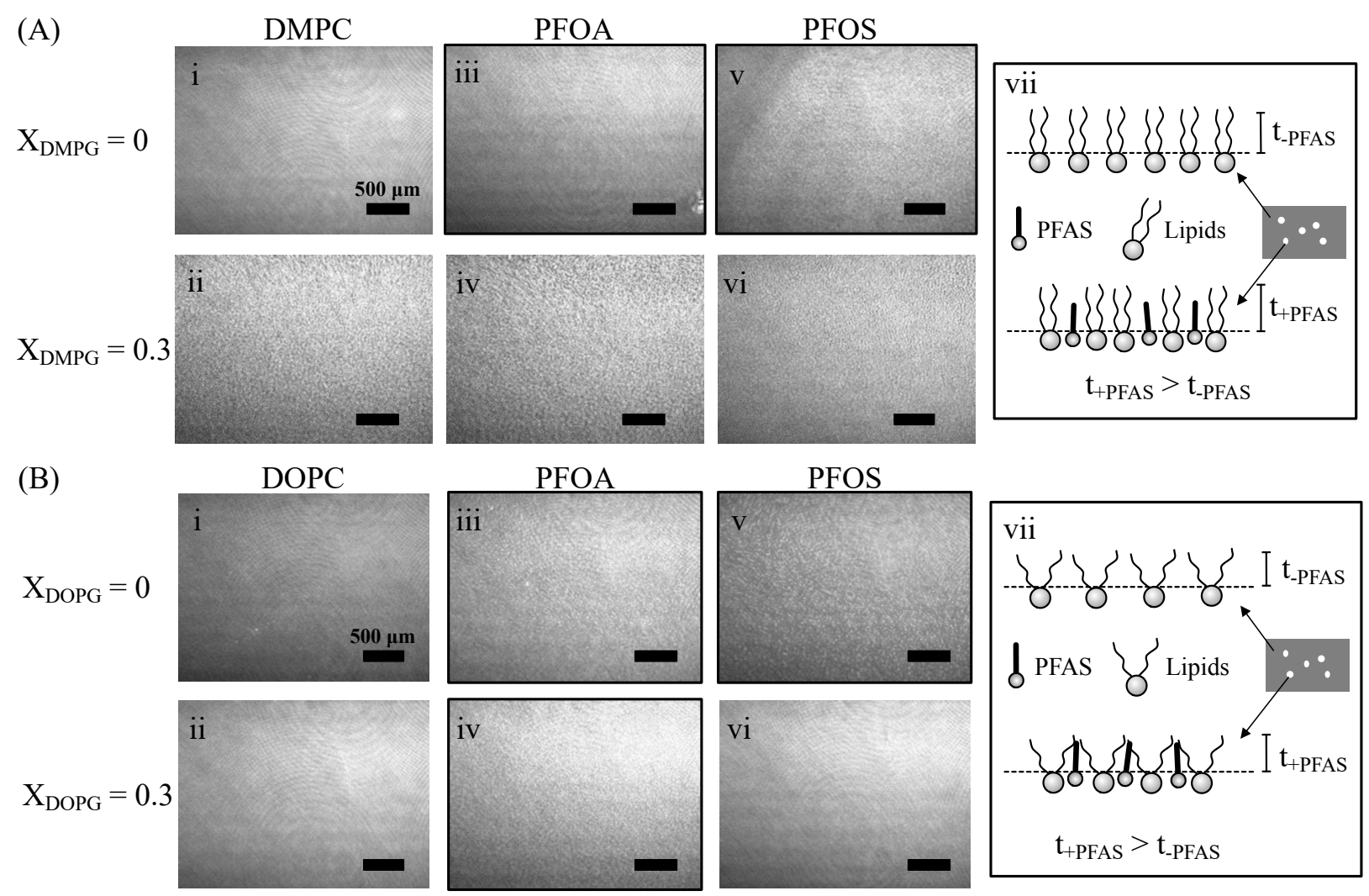

Figure 4. Brewster Angle Microscopy (BAM) images of (A) DMPC $\left(X_{\mathrm{DMPG}}=0 ; \mathrm{i}, \mathrm{iii}, \mathrm{v}\right)$ and

$274 \mathrm{DMPC} / \mathrm{DMPG}\left(\mathrm{X}_{\mathrm{DMPG}}=0.3\right.$; ii, iv, vi) monolayers at $40 \mathrm{mN} / \mathrm{m}$ and (B) DOPC ( $\mathrm{X}_{\mathrm{DOPG}}=0$; i, iii,

275 v) and DOPC/DOPG $\left(\mathrm{X}_{\mathrm{DOPG}}=0.3\right.$; ii, iv, vi) monolayers at $35 \mathrm{mN} / \mathrm{m}$ with added PFOA and

276 PFOS. The same scale is used for each image and monolayers exhibiting lipid condensation are

277 shown as images with black line border. Proposed schematics are shown (not to scale; vii)

278 depicting lipid condensation in the presence of PFAS. 
Viada et $\mathrm{al}^{40}$ have reported that perfluorodecanoic acid (PFDA) is expelled at high compression from anionic distearoylphosphatidic acid (DSPA) monolayers where the difference

282 in lipid tail length to $\mathrm{C}_{\mathrm{nF}}$ is 9 carbon atoms, but mixes within dilauroylphosphatidic acid (DLPA)

283 monolayers where the difference is 3 carbon atoms. DSPA and DLPA both contain saturated

284 tails. Comparatively, the tail length difference for PFOA and PFOS with DM lipids is 6 and 7

285 carbon atoms, respectively. This comparison shows that small changes in PFAS length - one

286 carbon atom - may determine whether PFAS are retained within or expelled from phospholipid

287 monolayers. Headgroup chemistry likely plays an important role as well and the ability for PFOS

288 to be retained within the monolayer could stem from stronger hydrogen bonding between the 289 sulfonic acid headgroup and the lipid headgroups.

291 3.2. Unsaturated Phospholipid Monolayers (DOPC and DOPC/DOPG)

292 The DOPC isotherm shows good agreement with the literature ${ }^{41}$ and with DOPG, 293 monotonic increases in $\pi$ are observed with compression with no evidence of LE-LC phase 294 transitions (expected for lipids with unsaturated tails; Figure 5a-c). The DOPC/DOPG isotherms 295 exhibit much of the same behavior observed for DMPC/DMPG, with greater lift-off areas with 296 increasing $\mathrm{X}_{\mathrm{DOPG}},\left(\sim 6 \%\right.$ from $\mathrm{X}_{\mathrm{DOPG}}=0$ to 0.3$)$ and, with PFAS present, PFOS having the 297 greatest effect on the isotherm. This similarity for lipids with short/saturated and 298 long/unsaturated tails shows the importance of electrostatic interactions between lipid and PFAS 299 headgroups.

300 The cis double bond in DOPC and DOPG tails leads to a fluid monolayer with low 301 packing densities compared to saturated lipids. This is reflected in the compressibility moduli 302 shown in Figure 5d-f. Interestingly, $\mathrm{C}_{\mathrm{s}}^{-1}$ for DOPC or DOPC/DOPG mixtures do not show 
303 significant differences without added PFAS. Both PFAS reduced $\mathrm{C}_{\mathrm{s}}^{-1}$ (increased compressibility) 304 compared to the pure lipid, however with added PFAS the $\mathrm{C}_{s}^{-1}$ profiles increased with increasing $305 \mathrm{X}_{\mathrm{DOPG}}$ (decreased compressibility). Compared to DMPC/DMPG, these results suggest that 306 DOPC/DOPG can more easily accommodate the PFAS with the void space provided by 307 unsaturated tails, reducing the impact of unfavorable electrostatic repulsion. Since PFAS 308 partitioned into these void spaces, there was less of an effect on the compressibility as the lipid 309 heads are spaced apart by the unsaturated lipid tails. The proposed mechanism is supported by 310 the differences in minimum area, showing that PFASs occupy a lower effective area compared to 311 DMPC/DMPG (Figure 2), and the $\pi_{\max }$ (Figure 3), showing that less PFAS is excluded from the 312 monolayer upon collapse or plateau. 

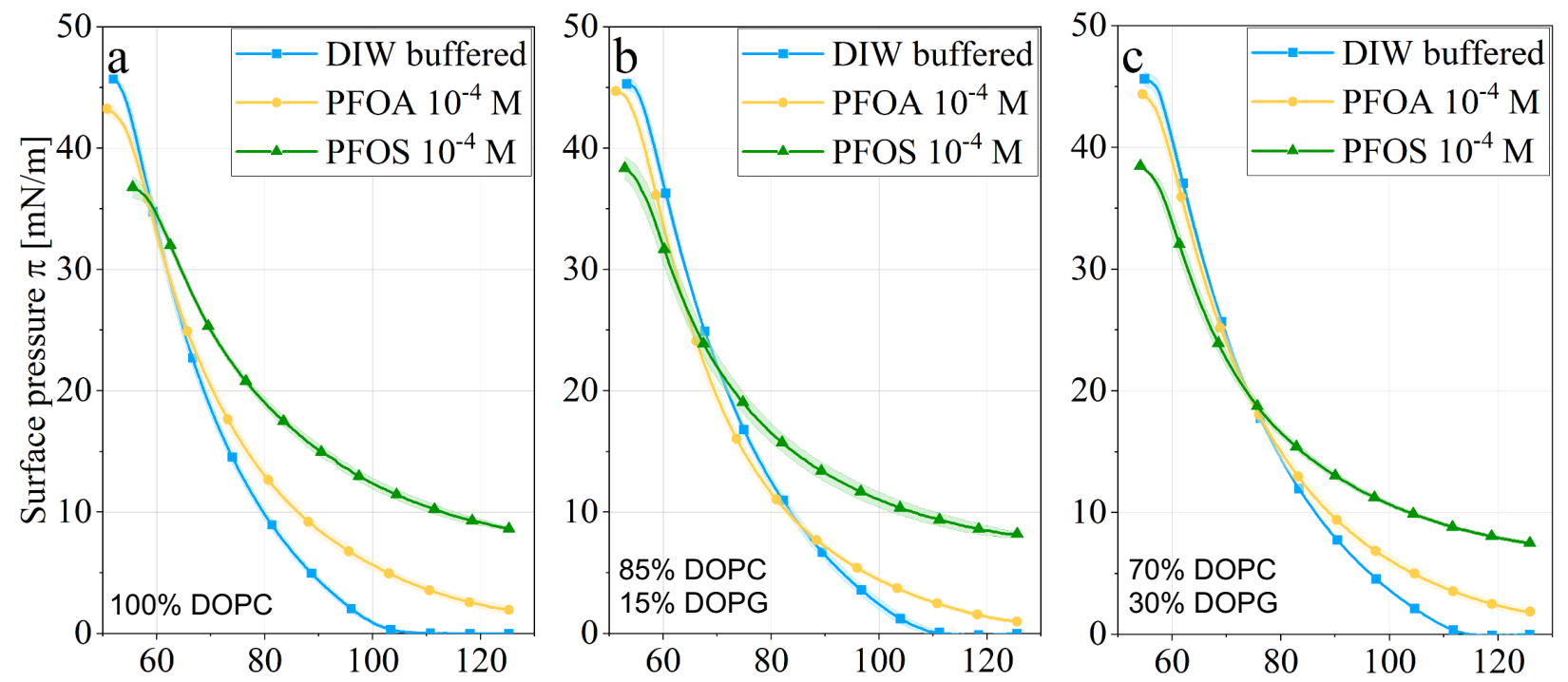

Mean molecular area $\overline{\mathrm{A}}\left[\AA^{2}\right]$

Mean molecular area $\overline{\mathrm{A}}\left[\AA^{2}\right]$

Mean molecular area $\bar{A}\left[\AA^{2}\right]$
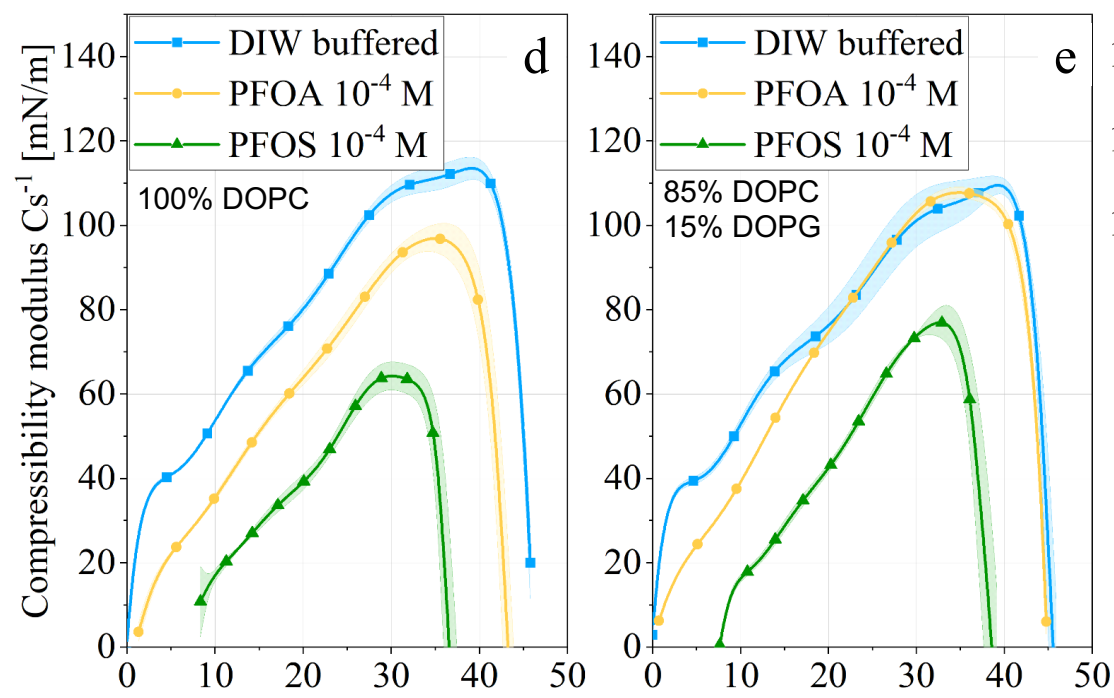

314

Surface pressure $\pi[\mathrm{mN} / \mathrm{m}]$

Surface pressure $\pi[\mathrm{mN} / \mathrm{m}]$

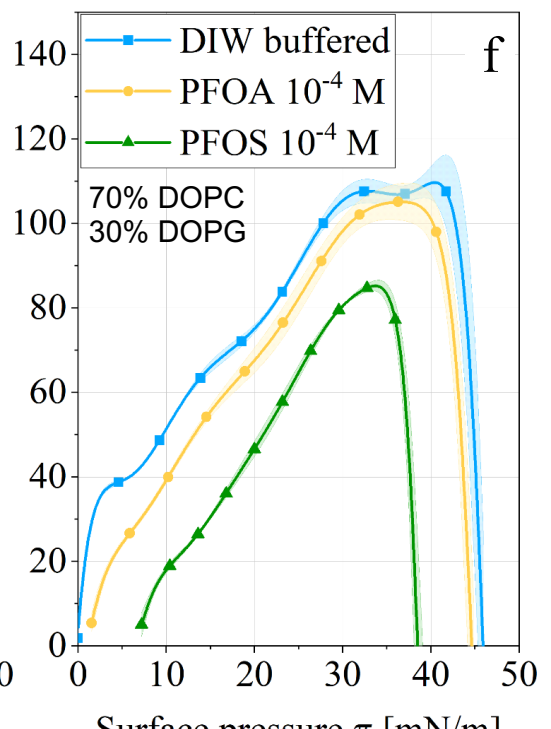

315 Figure 5. Surface pressure-area isotherms, $\pi-\bar{A}(a-c)$, and compressibility moduli, $C_{s}{ }^{-1}$ (d-f), for

316 DOPC monolayers as a function of DOPG concentration $\left(\mathrm{X}_{\mathrm{DOPG}}\right)$ in the absence (blue squares)

317 or presence of $10^{-4} \mathrm{M}$ PFOA (yellow circles) or PFOS (green triangles). a, d) $\mathrm{X}_{\mathrm{DOPG}}=0$; b, e)

$318 \mathrm{X}_{\mathrm{DOPG}}=0.15$; and $\left.\mathrm{c}, \mathrm{f}\right) \mathrm{X}_{\mathrm{DOPG}}=0.3$. The colored bands shown in a-f for each condition represent

319 the standard error of three independent experiments. 
Like DMPC, BAM images show the PFOA and PFOS can lead to condensed solid

322 domains in DOPC monolayers (Figure 4B) despite unfavorable lipid packing of the DOPC acyl

323 tails. There was also evidence of PFOA causing condensation in DOPC/DOPG $\left(\mathrm{X}_{\mathrm{DOPG}}=0.3\right)$

324 monolayers, which are not known to form LC domains. Experimental results for DOPC support

325 recent molecular dynamic simulations showing that PFOA and PFOS cause unsaturated lipids to 326 condense in a bilayer. ${ }^{42}$

327 To examine whether electrostatic repulsion would prevent PFAS partitioning into net 328 anionic monolayers, experiments were conducted where PFOS was added to the subphase with 329 preexisting $\mathrm{PC}$ and $\mathrm{PC} / \mathrm{PG}\left(\mathrm{X}_{\mathrm{PG}}=0.3\right)$ monolayers at the air/water interface and the relative 330 dynamic surface pressure, $\pi / \pi_{0}$ (initial surface pressure $\pi_{0}=35 \mathrm{mN} / \mathrm{m}$ ), was measured over $8 \mathrm{~h}$.

331 In all cases the addition of PFOS led to an increase in $\pi / \pi_{0}$ compared to the PFOS-free condition 332 over the duration of the experiments (Figure S6). This indicates that PFOS penetrated the 333 monolayer and led to increased lipid packing at the interface, which increased $\pi$ irrespective of 334 the net-charge of the monolayer or lipid tail saturation. The effect of PFOS on was less 335 pronounced for monolayers with unsaturated lipids, further supporting the proposed mechanism 336 of PFAS adsorption in void space at the interface.

\subsection{Extracted Bacterial Lipid Monolayers}

339 The major classes of lipids identified in the bacterial lipid extracts were

340 phosphatidylglycerols (PG; $46.8 \pm 0.5 \%$ ), phosphatidylethanolamine (PE; 33.7 $\pm 0.8 \%$ ), and

341 glyceroglycolipids (18.3 $\pm 0.2 \%)$. LysoPE and phosphatidic acid (PA) were also present at $0.6 \pm$

$3420.1 \%$ each. The abundant species of PE and PG lipids had a total of 32 or 34 carbon atoms; 32

343 carbon atoms likely correspond to lipids with two $\mathrm{C}_{16}$ tails or one $\mathrm{C}_{14}$ and one $\mathrm{C}_{18}$ tail, while 34 
344 carbon atoms likely corresponded to one $\mathrm{C}_{16}$ tail and one $\mathrm{C}_{18}$ tail. For both species there were 1

345 or 2 degrees of fatty acid tail unsaturation (double bonds). For comparison, DMPC contains two

346 saturated $\mathrm{C}_{14}$ tails and DOPC contains two $\mathrm{C}_{18}$ tails, each with a double bond between $\mathrm{C}_{9}=\mathrm{C}_{10}$

347 that leads to void space for PFAS adsorption within a monolayer.

For Langmuir trough studies on model bacterial lipid monolayers the range of PFAS was

349 expanded to include PFHxS $\left(\mathrm{C}_{\mathrm{nF}}=6\right)$ and PFNA $\left(\mathrm{C}_{\mathrm{nF}}=8\right)$, in addition to PFOS $\left(\mathrm{C}_{\mathrm{nF}}=8\right)$ and

350 PFOA $\left(\mathrm{C}_{\mathrm{nF}}=7\right)$. Bacterial lipid monolayer isotherms display properties of both DMPC/DMPG

351 and DOPC/DOPG. Without PFAS, a transition from a less-ordered to a more-ordered phase is

352 observed from $\sim 15-25 \mathrm{mN} / \mathrm{m}$ (Figure 6 ; the transition cannot be defined as LE-LC) and the

353 monolayer is highly compressible (low $\mathrm{C}_{\mathrm{s}}{ }^{-1}$; Figure $\mathrm{S} 7$ ).

354 With added PFASs, $\pi$ after initial monolayer formation increased with increasing PFAS

355 concentration and the monolayers were more fluid and compressible. The isotherms with PFNA

356 and PFOS are very similar (Figures $6 \mathrm{~b}$ and d, respectively), suggesting that PFASs interactions

357 within the monolayer are due to length of the fluorinated tail for these PFAS with $\mathrm{C}_{\mathrm{nF}}=8$ and

358 that the effect of the headgroup, carboxylic vs sulfonic acid, is small in comparison. This is

359 consistent with the similar surface activities measured for PFNA and PFOS (Figure S5). The

360 intersections of the isotherms are shifted to larger areas with increasing concentrations,

361 consistent with increased headgroup electrostatic repulsion that reflects the charge of PFAS and

362 the high concentration of negative PG lipids in the bacterial lipid monolayers.

363 Fluorinated tail length does not however explain the differences observed for PFOA and

364 PFHxS. With PFOA $\left(\mathrm{C}_{\mathrm{nF}}=7\right.$; Figure $\left.6 \mathrm{c}\right)$ there was almost no change in the isotherms, while the

365 shorter PFHxS $\left(\mathrm{C}_{\mathrm{nF}}=6\right.$; Figure 6a) led to an expanded and more compressible monolayer. The

366 less-ordered to more-ordered phase transition also became more pronounced with increasing 
had no effect.
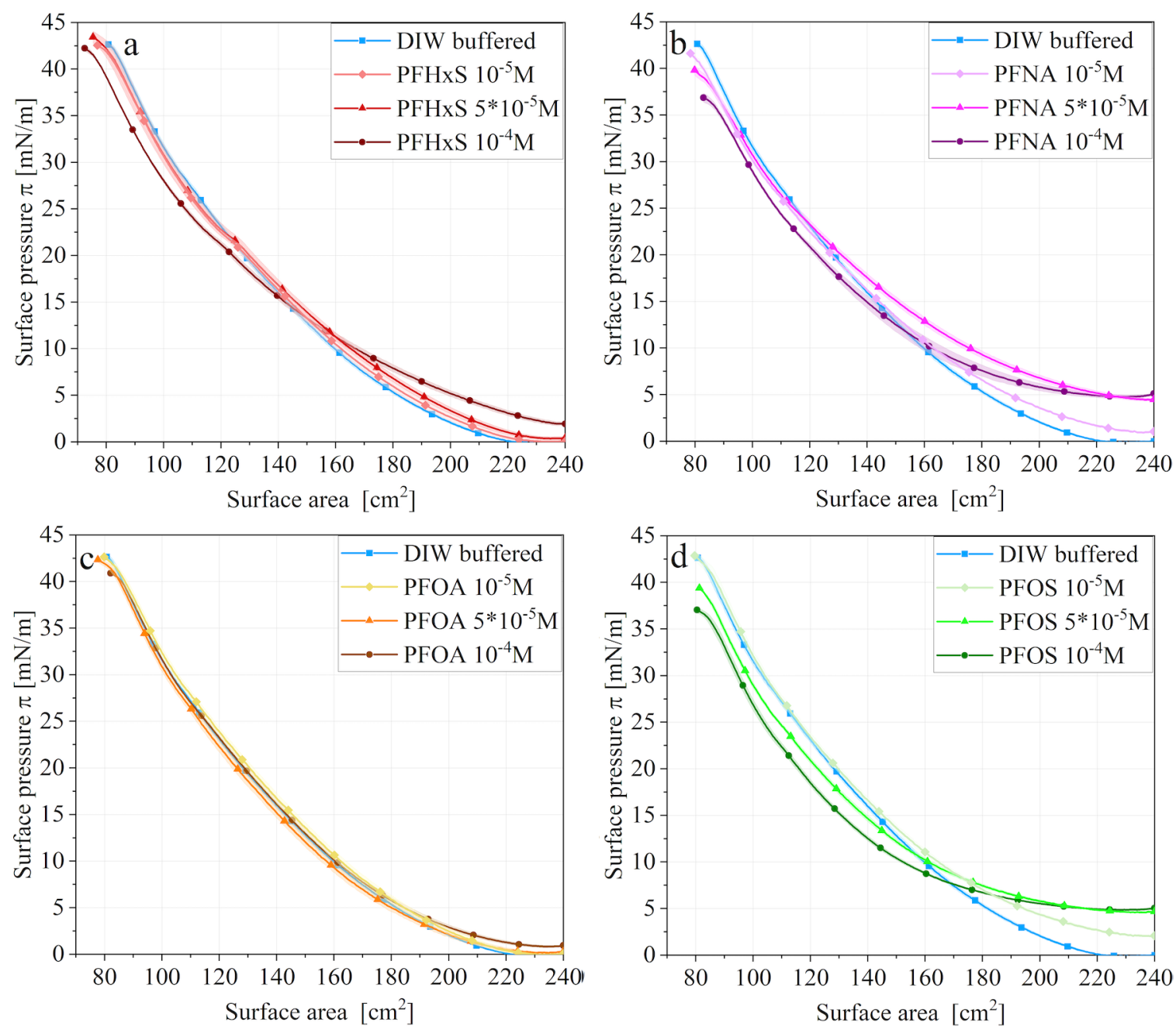

370 Figure 6. Surface pressure-area $(\pi-\mathrm{A})$ isotherms for extracted bacterial lipid monolayers in the

371 absence or presence of a) PFHxS, b) PFNA, c) PFOA, and d) PFOS as a function of PFAS

372 concentration. The colored bands shown in a-f for each condition represent the standard error of

373 three independent experiments.

374

PFHxS is an interesting molecule compared to similar PFAS. Albumin binding studies have shown that PFAS partition coefficients increase with increasing number of fluorinated 
377 carbons with the exception of PFHxS, which exhibited greater protein binding. ${ }^{43}$ The half-life of

378 PFHxS in humans is also greater than expected based on $\mathrm{C}_{\mathrm{nF}} .{ }^{44}$ These studies suggest that PFHxS

379 may exhibit more hydrophobic behavior than expected, which would explain its high surface

380 activity (Figure S5) despite its high water solubility compared to the other PFAS examined. ${ }^{33}$

381 Monolayer collapse occurs when a monolayer becomes tightly packed and unstable,

382 which leads to buckling or the formation of multilayer regions at a liquid interface. For bacterial

383 lipid monolayers, the surface pressure upon collapse decreased linearly with increasing PFOA,

384 PFNA, and PFOS concentrations in the subphase (Figure 7), with PFNA and PFOS being nearly

385 identical. A decrease in the collapse surface pressure reflects additional electrostatic repulsion

386 due to the presence of PFAS in the monolayer that prevents the lipids from packing as tightly at

387 the point of collapse. The collapse pressure is similar to DOPC/DOPG for these three PFAS at

$38810^{-4} \mathrm{M}$ (Figure 5). PFHxS is again an anomaly - despite fluidizing the monolayer, the collapse

389 pressure differed by just $\sim 0.4 \mathrm{mN} / \mathrm{m}$ from the control and did not change with PFHxS

390 concentration.

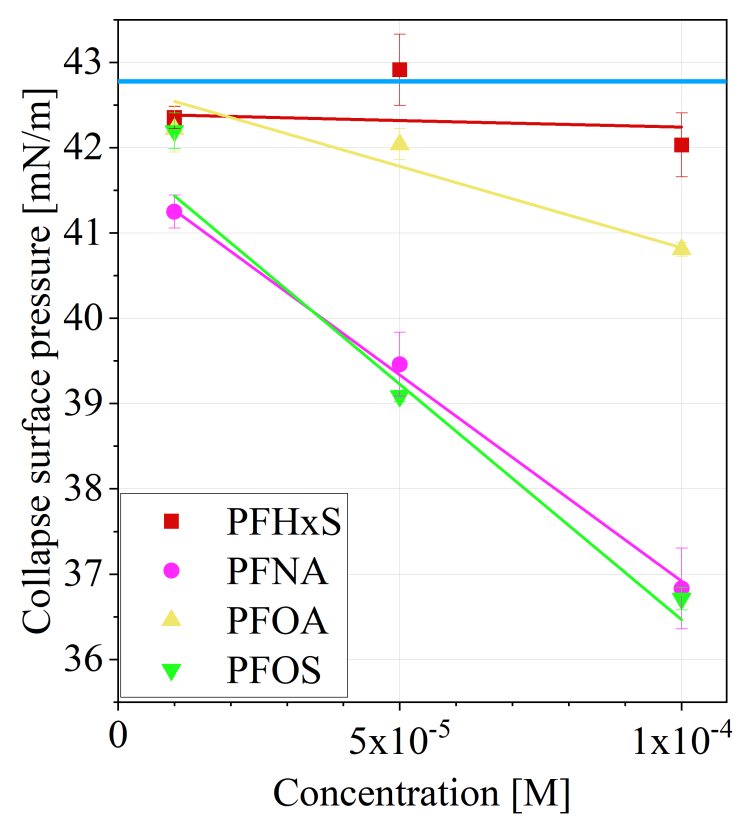


392 Figure 6. Collapse surface pressure of bacterial lipid monolayers as a function of PFAS

393 subphase concentration. The blue horizontal line is the collapse pressure at $42.8 \mathrm{mN} / \mathrm{m}$ of the

394 monolayers without PFAS. Standard error bars are shown based on triplicate measurements.

395 Error bars not visible are smaller than the symbols.

A clear dissimilarity between the bacterial and synthetic lipid monolayers was observed 398 when dynamic $\pi / \pi_{0}$ was measured for the bacterial lipid monolayers when PFAS was injected 399 into the subphase. PFOS as well as PFHxS, PFOA, and PFNA had little effect on $\pi$ over $8 \mathrm{~h}$, 400 suggesting that high content of negatively charged lipid (47.4 mol\% PG + PA) and unsaturated 401 lipid tails provided ample void space (high effective area per lipid) for PFAS to adsorb at the 402 air/water interface without packing the lipids. This was observed to a lesser extent for 403 DOPC/DOPG with increasing PG lipid content up to $30 \mathrm{~mol} \%$. There was no clear BAM 404 evidence of PFAS causing lipid condensation in the bacterial lipid monolayers.

\section{Conclusions} monolayers was dependent on the extent to which it was retained within the monolayer during

409 compression and the ability for the monolayer accommodate the PFAS in void spaces caused by 410 unsaturated lipids. PFOA was partially expelled from the monolayers at high compression where

411 the more hydrophobic PFOS was retained and contributed an additional repulsive interaction that 412 ultimately led to more fluid, compressible monolayer that collapsed with less force. By

413 combining BAM and isotherm results, experimental evidence confirmed prior computational 414 results showing that PFAS can cause phospholipid condensation. Interestingly, we observed that 
415 PFOA and PFOS caused lipid condensation while still yielding a more fluid, compressible 416 monolayer. Other molecules such as cholesterol that cause lipid condensation also increase the

417 rigidity and reduce the compressibility of lipid monolayers.

418 The effects of PFAS on the fluidity and compressibility of synthetic monolayers were

419 also observed for extracted bacterial membrane monolayers. For the bacterial lipid monolayers,

420 PFOS and PFNA, a sulfonic acid and a carboxylic acid both with $\mathrm{C}_{8}$ fluorinated tails, fluidized

421 the monolayers and led to early monolayer collapse. PFOA and PFHxS had comparably modest

422 effects. Given the thousands of PFAS present in our environment, additional studies are needed

423 to determine if the interactions observed in this work can be extended to classes of PFAS as well

424 as other PFAS structures (e.g. cationic PFAS or PFAS precursors), and if similar effects of are

425 observed for other environmentally relevant bacterial membranes.

426

\section{Acknowledgements}

428 This work was funded by the National Institute of Environmental Health Science

429 Sources, Transport, Exposure \& Effects of PFASs (STEEP) Superfund Research Program under 430 grant P42ES027706. STEEP is a partnership of the University of Rhode Island, the Harvard T.H.

431 Chan School of Public Health Department of Environmental Health, and the Silent Spring

432 Institute. The lipid analyses described in this work were performed at the Kansas Lipidomics

433 Research Center Analytical Laboratory. Instrument acquisition and lipidomics method

434 development were supported by the National Science Foundation (including support from the 435 Major Research Instrumentation program; most recent award DBI-1726527), K-IDeA Networks 436 of Biomedical Research Excellence (INBRE) of National Institute of Health (P20GM103418),

437 USDA National Institute of Food and Agriculture (Hatch/Multi-State project 1013013), and 
438 Kansas State University. We are grateful to Bongsup Cho, Daniel Roxbury, and Sigrid Berka for 439 their valuable insight.

440

441

\section{References}

442 (1) Ghisi, R.; Vamerali, T.; Manzetti, S. Accumulation of Perfluorinated Alkyl Substances 443 (PFAS) in Agricultural Plants: A Review. Environ. Res. 2019, 169, 326-341.

444 (2) Pérez, F.; Nadal, M.; Navarro-Ortega, A.; Fàbrega, F.; Domingo, J. L.; Barceló, D.; Farré, 445 M. Accumulation of Perfluoroalkyl Substances in Human Tissues. Environ. Int. 2013, 59, 446 354-362.

447 (3) Kwiatkowski, C. F.; Andrews, D. Q.; Birnbaum, L. S.; Bruton, T. A.; DeWitt, J. C.;

Knappe, D. R. U.; Maffini, M. V.; Miller, M. F.; Pelch, K. E.; Reade, A.; et al. Scientific

(4) Fiedler, S.; Pfister, G.; Schramm, K.-W. Poly- and Perfluorinated Compounds in Basis for Managing PFAS as a Chemical Class. Environ. Sci. Technol. Lett. 2020, 7 (8), Household Consumer Products. Toxicol. Environ. Chem. 2010, 92 (10), 1801-1811.

(5) Kotthoff, M.; Müller, J.; Jürling, H.; Schlummer, M.; Fiedler, D. Perfluoroalkyl and (19), 14546-14559.

(6) Fujii, S.; Polprasert, C.; Tanaka, S.; Hong Lien, N. P.; Qiu, Y. New POPs in the Water Environment: Distribution, Bioaccumulation and Treatment of Perfluorinated Compounds - a Review Paper. J. Water Supply Res. Technol. 2007, 56 (5), 313-326.

(7) Huang, R.; Chen, Q.; Zhang, L.; Luo, K.; Chen, L.; Zhao, S.; Feng, L.; Zhang, J. Prenatal 
Disorders of Pregnancy. Environ. Heal. 2019, 18 (1), 5.

462

463

464

465

466

467

468

469

470

471

472

473

474

475

476

477

478

479

480

481

482

483

(8) Post, G. B.; Gleason, J. A.; Cooper, K. R. Key Scientific Issues in Developing Drinking Water Guidelines for Perfluoroalkyl Acids: Contaminants of Emerging Concern. PLOS Biol. 2017, 15 (12), e2002855.

(9) Rotander, A.; Toms, L.-M. L.; Aylward, L.; Kay, M.; Mueller, J. F. Elevated Levels of PFOS and PFHxS in Firefighters Exposed to Aqueous Film Forming Foam (AFFF). Environ. Int. 2015, 82, 28-34.

(10) Reth, M.; Berger, U.; Broman, D.; Cousins, I. T.; Nilsson, E. D.; McLachlan, M. S. Water-to-Air Transfer of Perfluorinated Carboxylates and Sulfonates in a Sea Spray Simulator. Environ. Chem. 2011, 8 (4), 381.

(11) Kwok, K. Y.; Yamazaki, E.; Yamashita, N.; Taniyasu, S.; Murphy, M. B.; Horii, Y.; Petrick, G.; Kallerborn, R.; Kannan, K.; Murano, K.; et al. Transport of Perfluoroalkyl Substances (PFAS) from an Arctic Glacier to Downstream Locations: Implications for Sources. Sci. Total Environ. 2013, 447, 46-55.

(12) Dassuncao, C.; Pickard, H.; Pfohl, M.; Tokranov, A. K.; Li, M.; Mikkelsen, B.; Slitt, A.; Sunderland, E. M. Phospholipid Levels Predict the Tissue Distribution of Poly- and Perfluoroalkyl Substances in a Marine Mammal. Environ. Sci. Technol. Lett. 2019, 6 (3), 119-125.

(13) Ng, C. A.; Hungerbühler, K.; Hungerbuhler, K. Bioaccumulation of Perfluorinated Alkyl Acids: Observations and Models. Env. Sci Technol 2014, 48 (9), 4637-4648.

(14) Ng, C. A.; Hungerbühler, K. Bioconcentration of Perfluorinated Alkyl Acids: How Important Is Specific Binding? Environ. Sci. Technol. 2013, 47 (13), 7214-7223.

(15) Ngyugen, H.; McNamee, C. E. Determination and Comparison of How the Chain Number 
and Chain Length of a Lipid Affects Its Interactions with a Phospholipid at an Air/Water Interface. J. Phys. Chem. B 2014, 118 (22), 5901-5912.

486

487

488

489

490

491

492

493

494

495

496

497

502

503

504

505

506

(16) Xie, W.; Ludewig, G.; Wang, K.; Lehmler, H.-J. J. Model and Cell Membrane

Partitioning of Perfluorooctanesulfonate Is Independent of the Lipid Chain Length.

Colloids and Surfaces B-Biointerfaces 2010, 76 (1), 128-136.

(17) Nouhi, S.; Ahrens, L.; Campos Pereira, H.; Hughes, A. V.; Campana, M.; Gutfreund, P.; Palsson, G. K.; Vorobiev, A.; Hellsing, M. S. Interactions of Perfluoroalkyl Substances with a Phospholipid Bilayer Studied by Neutron Reflectometry. J. Colloid Interface Sci. 2018, 511, 474-481.

(18) Oldham, E. D.; Xie, W.; Farnoud, A. M.; Fiegel, J.; Lehmler, H.-J. J. Disruption of Phosphatidylcholine Monolayers and Bilayers by Perfluorobutane Sulfonate. J Phys Chem B 2012, 116 (33), 9999-10007.

(19) Matyszewska, D.; Bilewicz, R. Influence of Perfluorinated Compounds on Model Lipid Membranes Prepared Using Langmuir and Langmuir-Schaefer Techniques. Colloids Surfaces A Physicochem. Eng. Asp. 2008, 321 (1-3), 11-15.

(20) Matyszewska, D.; Tappura, K.; Orädd, G.; Bilewicz, R. Influence of Perfluorinated Compounds on the Properties of Model Lipid Membranes. J. Phys. Chem. B 2007, 111 (33), 9908-9918.

(21) Lehmler, H.-J. J.; Xie, W.; Bothun, G. D.; Bummer, P. M.; Knutson, B. L. Mixing of Perfluorooctanesulfonic Acid (PFOS) Potassium Salt with Dipalmitoyl Phosphatidylcholine (DPPC). Coll. Surf. B 2006, 51 (1), 25-29.

(22) Xie, W.; Bothun, G. D.; Lehmler, H.-J. J. Partitioning of Perfluorooctanoate into Phosphatidylcholine Bilayers Is Chain Length-Independent. Chem. Phys. Lipids 2010, 163 
(3), 300-308.

508

509

510

511

(23) Fitzgerald, N. J. M.; Wargenau, A.; Sorenson, C.; Pedersen, J.; Tufenkji, N.; Novak, P. J.; Simcik, M. F. Partitioning and Accumulation of Perfluoroalkyl Substances in Model Lipid Bilayers and Bacteria. Environ. Sci. Technol. 2018, 52 (18), 10433-10440.

(24) Fitzgerald, N. J. M.; Simcik, M. F.; Novak, P. J. Perfluoroalkyl Substances Increase the Membrane Permeability and Quorum Sensing Response in Aliivibrio Fischeri. Environ. Sci. Technol. Lett. 2018.

(25) Liu, G.; Zhang, S.; Yang, K.; Zhu, L.; Lin, D. Toxicity of Perfluorooctane Sulfonate and Perfluorooctanoic Acid to Escherichia Coli: Membrane Disruption, Oxidative Stress, and DNA Damage Induced Cell Inactivation and/or Death. Environ. Pollut. 2016.

(26) Weathers, T. S.; Higgins, C. P.; Sharp, J. O. Enhanced Biofilm Production by a TolueneDegrading Rhodococcus Observed after Exposure to Perfluoroalkyl Acids. Environ. Sci. Technol. 2015, 49 (9), 5458-5466.

(27) Yakimov, M. M.; Golyshin, P. N.; Lang, S.; Moore, E. R. B.; Abraham, W.-R.; Lunsdorf, H.; Timmis, K. N. Alcanivorax Borkumensis Gen. Nov., Sp. Nov., a New, HydrocarbonDegrading and Surfactant-Producing Marine Bacterium. Int. J. Syst. Bacteriol. 1998, 48 (2), 339-348.

(28) Schneiker, S.; dos Santos, V. A. M.; Bartels, D.; Bekel, T.; Brecht, M.; Buhrmester, J.; Chernikova, T. N.; Denaro, R.; Ferrer, M.; Gertler, C.; et al. Genome Sequence of the Ubiquitous Hydrocarbon-Degrading Marine Bacterium Alcanivorax Borkumensis. Nat. Biotechnol. 2006, 24 (8), 997-1004.

(29) Konieczna, M.; Olzog, M.; Naether, D.; Chrzanowski, Ł.; Heipieper, H. Membrane Fatty Acid Composition and Cell Surface Hydrophobicity of Marine Hydrocarbonoclastic 
Alcanivorax Borkumensis SK2 Grown on Diesel, Biodiesel and Rapeseed Oil as Carbon Sources. Molecules 2018, 23 (6), 1432.

USEPA. CompTox Chemicals Dashboard https://comptox.epa.gov/dashboard/.

533

(31) Anaya, N. M.; Faghihzadeh, F.; Ganji, N.; Bothun, G.; Oyanedel-Craver, V. Comparative

Study between Chemostat and Batch Reactors to Quantify Membrane Permeability Changes on Bacteria Exposed to Silver Nanoparticles. Sci. Total Environ. 2016, 565, 841848.

(32) Bligh, E. G.; Dyer, W. J. A Rapid Method of Total Lipid Extraction and Purification. Can. J. Biochem. Physiol. 1959, 37 (8), 911-917.

(33) Kim, M.; Li, L. Y.; Grace, J. R.; Yue, C. Selecting Reliable Physicochemical Properties of Perfluoroalkyl and Polyfluoroalkyl Substances (PFASs) Based on Molecular Descriptors. Environ. Pollut. 2015, 196 (May 2009), 462-472.

(34) Schaefer, C. E.; Culina, V.; Nguyen, D.; Field, J. Uptake of Poly- and Perfluoroalkyl Substances at the Air-Water Interface. Environ. Sci. Technol. 2019, 53 (21), 1244212448

(35) Costanza, J.; Arshadi, M.; Abriola, L. M.; Pennell, K. D. Accumulation of PFOA and PFOS at the Air-Water Interface. Environ. Sci. Technol. Lett. 2019, 6 (8), 487-491.

(36) Park, C. K.; Schmitt, F. J.; Evert, L.; Schwartz, D. K.; Israelachvili, J. N.; Knobler, C. M. Film Balance and Fluorescence Microscopic Investigation of the Effects of $\mathrm{Ca} 2+$ on Mixed DMPC/DMPG Monolayers. Langmuir 1999, 15 (1), 202-206.

(37) Vernoux, N.; Maniti, O.; Besson, F.; Granjon, T.; Marcillat, O.; Vial, C. Mitochondrial Creatine Kinase Adsorption to Biomimetic Membranes: A Langmuir Monolayer Study. J. Colloid Interface Sci. 2007, 310 (2), 436-445. 
553 (38) Boggs, J. M. Lipid Intermolecular Hydrogen Bonding: Influence on Structural Organization and Membrane Function. Biochim. Biophys. Acta - Rev. Biomembr. 1987, $906(3), 353-404$.

(39) Lv, G.; Sun, X. The Molecular-Level Understanding of the Uptake of PFOS and Its Alternatives (6:2 Cl-PFESA and OBS) into Phospholipid Bilayers. J. Hazard. Mater. 2021, 417, 125991.

(40) Viada, B.; Cámara, C. I.; Yudi, L. M. Destabilizing Effect of Perfluorodecanoic Acid on Simple Membrane Models. Soft Matter 2019, 15 (11), 2447-2462.

(41) Guzmán, E.; Liggieri, L.; Santini, E.; Ferrari, M.; Ravera, F. DPPC-DOPC Langmuir Monolayers Modified by Hydrophilic Silica Nanoparticles: Phase Behaviour, Structure and Rheology. Colloids Surfaces A Physicochem. Eng. Asp. 2012, 413, 174-183.

(42) Shen, Z.; Ge, J.; Ye, H.; Tang, S.; Li, Y. Cholesterol-like Condensing Effect of

(43) Allendorf, F.; Berger, U.; Goss, K.-U. U.; Ulrich, N. Partition Coefficients of Four Perfluoroalkyl Acid Alternatives between Bovine Serum Albumin (BSA) and Water in $21(11), 1852-1863$.

Olsen, G. W.; Burris, J. M.; Ehresman, D. J.; Froehlich, J. W.; Seacat, A. M.; Butenhoff, J. L.; Zobel, L. R. Half-Life of Serum Elimination of Perfluorooctanesulfonate, Perfluorohexanesulfonate, and Perfluorooctanoate in Retired Fluorochemical Production Workers. Environ. Health Perspect. 2007, 115 (9), 1298-1305.

575 